\title{
Magnetized Mars: Spatial distribution of oxygen ions
}

\author{
Esa Kallio ${ }^{1}$ and Stas Barabash ${ }^{2}$ \\ ${ }^{1}$ Finnish Meteorological Institute, Helsinki, Finland \\ ${ }^{2}$ Swedish Institute of Space Physics, Kiruna, Sweden \\ (Received February 4, 2011; Revised June 26, 2011; Accepted July 13, 2011; Online published March 8, 2012)
}

\begin{abstract}
We have studied the spatial distribution of oxygen ions near Mars assuming that the planet had a weak intrinsic magnetic field sometimes in the past. The study has been performed by using a global self-consistent numerical hybrid model HYB-Mars by simulating four magnetization cases when the strength of the dipole magnetic field on surface at the magnetic equator was $0 \mathrm{nT}, 10 \mathrm{nT}, 30 \mathrm{nT}$ and $60 \mathrm{nT}$. In all cases the upstream solar wind conditions were assumed to be present day nominal values. Two different regions were found: (1) a closed magnetic field line region where the density of oxygen ions was high and the ion velocity small and (2) an open magnetic region near the magnetic poles where both the density and the velocity of planetary oxygen ions were high. The former region has similarities with Earth's plasmasphere and the latter with Earth's magnetic cusps. The size of the closed magnetic field region increases with increasing dipolar field. The oxygen ions originating from the ionosphere were found to escape easily along the magnetic field from the magnetic cusps but become trapped within the closed magnetic field line region. The model used does not include a self-consistent ionosphere but it is interesting to note that the total loss rate had a local maximum at a small non-zero value of the magnetic dipole field (10 nT).
\end{abstract}

Key words: Mars, solar wind, magnetospheres, magnetic field, evolution, numerical simulation.

\section{Introduction}

The present "magnetized Mars" study where Mars is assumed to has a small intrinsic magnetic has been inspired by two important observations. First, planetary oxygen ions are currently measured in detail by the ASPERA-3 instrument on ESA's Mars Express (MEX) mission. The measurements have provided global maps of the spatial distribution of the non-thermally escaping planetary $\mathrm{O}^{+}$and $\mathrm{O}_{2}{ }^{+}$ ions and their energization when the solar wind accelerates planetary ions above the escape velocity (see, e.g. Barabash et al., 2007). Secondly, Mars Global Surveyor (MGS) detected strong $(>1000 \mathrm{nT})$ localized magnetized regions at Mars (Acuña et al., 1998, 2001; Connerney et al., 2004) suggesting that in the past Mars may have had a strong global intrinsic magnetic dynamo which has thereafter decayed.

These observations prompt one to ask how the global intrinsic magnetic field at Mars may have affected the interaction between Martian upper atmosphere and the Solar wind. That is a fundamental issue if one wants to understand the evolution of the Martian atmosphere. Moreover, the role the planetary global intrinsic magnetic field plays in the planetsolar wind interactions is of a more general interest, because it is also linked to the escape of oxygen ions from Earth (see, e.g. Moore and Khazanov, 2010), as well as from Earth-like planets in our and other Solar Systems.

Global numerical simulations provide a tool to study

Copyright (C) The Society of Geomagnetism and Earth, Planetary and Space Sciences (SGEPSS); The Seismological Society of Japan; The Volcanological Society of Japan; The Geodetic Society of Japan; The Japanese Society for Planetary Sciences; TERRAPUB.

doi:10.5047/eps.2011.07.008 self-consistently how planetary ions interact with the solar wind and its electromagnetic fields. In practice, however, a three dimensional planet-solar wind interaction model has to adopt various approximations and simplifications because physical processes of interest cover a wide time and space range (see, for example, discussion in Ledvina et al., 2008). This is the case for Earth (see the recent review of $\mathrm{O}^{+}$escape modeling from Moore and Khazanov, 2010; Moore et al., 2010) and also for Mars (see comparison of seven global Mars-solar wind interaction models from Brain et al., 2010).

Although more detailed Mars-solar wind interaction models are required to study the role of the planetary magnetic field already current 3-D models are anticipated to bring new insight to the magnetized planet-solar wind interaction. However, this requires carefully planned simulations and a careful interpretation of the results. Recently, the morphology of the slightly magnetized Mars has been studied by a hybrid model, HYB-Mars (Kallio et al., 2008). The study showed how the Martian present day Venus-like "induced" magnetosphere transforms to an Earth-like magnetosphere when the Martian global intrinsic magnetic field at the equator was increased from $0 \mathrm{nT}$ to $10 \mathrm{nT}, 30 \mathrm{nT}$ and $60 \mathrm{nT}$. The simulation also suggested how the Martian possible past Earth-like magnetosphere has transformed to the present Venus-like induced magnetosphere when the Martian magnetic dynamo has decayed.

In the present work we continue to analyze magnetized Mars with the HYB-Mars model by studying the global spatial distribution of $\mathrm{O}^{+}$and $\mathrm{O}_{2}{ }^{+}$ions when Martian magnetic field is again $0 \mathrm{nT}$ to $10 \mathrm{nT}, 30 \mathrm{nT}$ and $60 \mathrm{nT}$ at the equator. The goal is to analyze how the previously studied Martian 
magnetic field magnetosphere cases (Kallio et al., 2008) are correlated with the properties of planetary oxygen ions.

The paper is organized as follow. First, the basic properties of the hybrid model are described. Then the density of $\mathrm{O}_{2}{ }^{+}$and $\mathrm{O}^{+}$ions near Mars is analyzed for the four intrinsic magnetic field cases. Finally, the implications of the results for the evolution of the atmospheres of Earth-like planets are discussed.

\section{Hybrid Model}

The global self-consistent hybrid model HYB-Mars developed at the Finnish Meteorological institute has been used in the simulations. In a hybrid model ions are modeled as particles while electrons form a massless charge neutralizing fluid. Detailed description of the HYB-Mars model is given elsewhere (Kallio et al., 2010), here we only describe its features relevant for the present study.

\subsection{Basic features}

The coordinate system and the orientation has been the same as in our previous magnetized Mars HYB-Mars study (Kallio et al., 2008): The $x$-axis points from the center of Mars to the Sun, the $z$-axis is perpendicular to the orbital plane of Mars pointing toward the ecliptic North and the $y$ axis completes the right hand coordinate system. The Martian intrinsic magnetic field is modeled as a magnetic dipole which is aligned with the $z$-axis and generates $\left[0,0, B_{\text {equator }}\right]$ magnetic field on the surface of Mars on the $z=0$ plane. As in the previous magnetized Mars study (Kallio et al., 2008 ) four $B_{\text {equator }}$ cases were compared: $0 \mathrm{nT}$ to $10 \mathrm{nT}$, $30 \mathrm{nT}$ and $60 \mathrm{nT}$. The dipolar magnetic field is on the $z-$ axis and, therefore, in the non-zero magnetic dipole case the North magnetic pole is on the North hemisphere at the point $\left(0,0, R_{\mathrm{M}}\right)$ and the South magnetic pole on the South hemisphere at $\left(0,0,-R_{\mathrm{M}}\right)$ where is the radius of Mars $(=3393$ $\mathrm{km})$.

The size of the simulation box was $-21,600 \mathrm{~km}<x, y$, $z<21,600 \mathrm{~km}$, approximately $-6 R_{\mathrm{M}}<x, y, z<6 R_{\mathrm{M}}$. The inner boundary in the model representing the exobase was a spherical boundary at $3600 \mathrm{~km}$ from the center of Mars, that is, about $200 \mathrm{~km}$ above Martian surface. In these simulations an ion was taken away from the simulation if it hit either the inner spherical boundary or the faces of the simulation box. The grid is divided into three concentric zones with grid spacing increasing outwards. The three grid sizes were $0.2 R_{\mathrm{M}}, 0.1 R_{\mathrm{M}}$ and $0.05 R_{\mathrm{M}}$ when the distance from the center of Mars $(r)$ was $r>3 R_{\mathrm{M}}, 2 R_{\mathrm{M}}<r<3 R_{\mathrm{M}}$ and $r<2 R_{\mathrm{M}}$, respectively.

The solar wind parameters were assumed to be close to the present day nominal parameters: The solar wind density $\left(n_{\mathrm{sw}}\right)$, the solar wind velocity $\left(\mathbf{U}_{\mathrm{sw}}\right)$ and the interplanetary magnetic field $\left(\mathbf{B}_{\mathrm{sw}}\right)$ were assumed to be $3 \mathrm{~cm}^{-3}$, $[-450,0,0] \mathrm{km} \mathrm{s}^{-1}$ and $\left[\cos \left(55^{\circ}\right),-\sin \left(55^{\circ}\right), 0\right] \times 1.12 \mathrm{nT}$ $=[0.6424,-0.9175,0] \mathrm{nT}$, respectively. The proton inertial length $\left(=c / \omega_{\mathrm{pi}}\right.$, where $c$ is the speed of light and $\omega_{\mathrm{pi}}$ is the plasma frequency of protons) in the solar wind is $\sim 130$ $\mathrm{km}$. It is noteworthy that the convective electric field in the solar wind, $\mathbf{E}_{\mathrm{sw}}\left(=-\mathbf{U}_{\mathrm{sw}} \times \mathbf{B}_{\mathrm{sw}}\right)$, points in this situation to the $-z$ direction. These parameters were identical with the values used in Kallio et al. (2008) study enabling a comparison of the results in the previous study and in the current study.

The model contains three ion species: $\mathrm{H}^{+}, \mathrm{O}^{+}$and $\mathrm{O}_{2}{ }^{+}$ ions. There are two $\mathrm{H}^{+}$sources in the simulation, (1) the solar wind $\mathrm{H}^{+}$ions and (2) $\mathrm{H}^{+}$ions formed from the Martian hydrogen corona by photoionization, charge exchange and electron impact ionization. There are also two $\mathrm{O}^{+}$ion populations: (1) $\mathrm{O}^{+}$ions formed from the Martian oxygen corona by photoionization, charge exchange and electron impact ionization and (2) $\mathrm{O}^{+}$ions escaping through the model exobase. All $\mathrm{O}_{2}{ }^{+}$ions escape through the model exobase. It is important to note that the model does not contain a self-consistent ionosphere and, therefore, the loss of $\mathrm{O}^{+}$and $\mathrm{O}_{2}{ }^{+}$ions from the ionosphere is taken into account in the model by injecting $\mathrm{O}^{+}$and $\mathrm{O}_{2}{ }^{+}$ions from the inner boundary into the simulation box. In other words, the HYB-Mars does not model physical processes which cause $\mathrm{O}^{+}$and $\mathrm{O}_{2}{ }^{+}$ion flux into the exosphere. In the simulation the $\mathrm{O}^{+}$and $\mathrm{O}_{2}{ }^{+}$injection flux at the dayside exobase was assumed to have Solar Zenith Angle (SZA) dependence of the form $\cos (\mathrm{SZA})$ and a homogenous flux on the nightside which corresponds $10 \%$ of the flux at the subsolar point. The initial temperatures of ionospheric $\mathrm{O}^{+}$and $\mathrm{O}_{2}+$ ions were assumed to be $10^{5} \mathrm{~K}$ and $\mathrm{O}^{+}$ions from the neutral corona $6.5 \times 10^{3} \mathrm{~K}$. All model parameters were kept similar in the four runs except the strength of the dipolar magnetic field.

\subsection{Limitations of the model}

Although the numerical hybrid simulation provides a self-consistent approach for studying the properties of planetary plasma environments it is important to note the following limitations and adopted simplifications when the results are interpreted. First, the upstream parameters represent nominal current day values, although the solar wind parameters have been proposed to be much different several Gyr ago (e.g. Wood, 2006). The adopted present day upstream parameters, however, make it possible to compare the known present day non-magnetized Mars $\mathrm{O}^{+}$and $\mathrm{O}_{2}{ }^{+}$ plasma environments with the magnetized Mars situation, isolating only for effects related to the planetary magnetic field.

Second, the adopted global intrinsic magnetic field is very weak even in the maximum intrinsic magnetic field case (60 nT at the magnetic equator on the surface) compared, for example, to the situation at the Earth $(\sim 30 \mu \mathrm{T}$ at the magnetic equator on the surface). It is, however, worth noting that, the size of the Martian magnetosphere is determined by a pressure balance between the upstream parameters and the intrinsic magnetic field which both are in the presented simulations smaller than they were several Gyr ago.

Third, Mars was modeled as a non-rotating planet and the angle between the magnetic dipole and the Mars-Sun line was assumed to be $90^{\circ}$, that is, the tilt angle is assumed to be zero. It is foreseen that non-zero tilt angle affects the size of the magnetic cusps, their connection to the IMF and, as seen later in this paper (Section 3.3) the leakage of the planetary ions into the solar wind. Including planetary rotation will complicate the situation even more.

Furthermore, the hybrid model does not include an ionosphere and, therefore, it cannot model effects in the iono- 
sphere caused by the intrinsic magnetic field. However, no fully self-consistent global model exists which could take into account various effects which play a role at the oxygen escape from the Earth-type magnetized ionosphere such as plasma waves, precipitating electrons, rotation of the planet, ion-neutral interactions and the conductivity of the ionosphere (see, for example, Moore and Khazanov, 2010).

Finally, the emission of protons and oxygen ions from the exosphere and the ionosphere resemble the present day EUV conditions. It has been argued that the coronal Xray-EUV emissions of the young main-sequence Sun were 100-1000 times stronger than those of the present Sun (Ribas et al., 2005). Therefore, the Martian ionosphere, the density of the hydrogen-oxygen neutral corona and the amount of planetary ions near young Mars are also anticipated to differ from the situation modelled in this paper.

\section{Results}

In this section we first study the spatial distribution of planetary $\mathrm{O}_{2}{ }^{+}$ions (Section 3.1). The density of $\mathrm{O}_{2}{ }^{+}$ions is closely linked with the morphology of the magnetic field which is analyzed in the following Section 3.2. Then the flow of $\mathrm{O}_{2}^{+}$and $\mathrm{O}^{+}$ions are investigated (Section 3.3). Finally, the ion loss rates are examined (Section 3.5).

\subsection{Spatial distribution of oxygen ions}

Figure 1 shows the density of $\mathrm{O}_{2}{ }^{+}$ions near Mars on the $y=0$ and $z=0$ planes in the four runs. The density is a time average over $25 \mathrm{~s}$. In the non-magnetized case (Fig. 1(a)) the density is highest near Mars on the North $(z>0)$ hemisphere. The non-zero $\mathrm{O}_{2}{ }^{+}$density region extends further from Mars on the South hemisphere $(z>0)$ than on the North hemisphere because the convective electric field accelerates planetary ions toward the $-z$ direction.

Comparison of the magnetized cases shows how increasing dipole field strength extends the spread of $\mathrm{O}_{2}+$ ions (Fig. 1(b-d)). Moreover, in the magnetized runs a clear increased density can be seen near the surface at midnight. A similar density increase can be found also near the subsolar point in the $30 \mathrm{nT}$ and $60 \mathrm{nT}$ cases. Note also the separate density increase on the Southern hemisphere $(z<0)$ in the solar wind in the $30 \mathrm{nT}$ and $60 \mathrm{nT}$ cases.

Figures $1(\mathrm{e}-\mathrm{h})$ give the $\mathrm{O}_{2}{ }^{+}$densities on the equator plane $(z=0)$. In the non-magnetized run two high density regions are formed on the night side (Fig. 1(e)). In the magnetized cases the high density region can be found further from the surface than in the non-magnetized case. Moreover, clear dawn $(y<0)$ to dusk $(y>0)$ asymmetry can be identified. On the dusk side the density forms a clear distinctive region where there are two areas of higher density, one near Mars and another "wing-like" density enhancement further from the planet. On the dawn side high density "patches" can be found and the $\mathrm{O}_{2}{ }^{+}$ions are not so clearly separated from the solar wind as on the dusk side. As will be seen later in Section 3.2, the properties of the planetary ion regions are closely related to the morphology of the global magnetic field.

\subsection{Plasma v.s. magnetic field regions}

Figure 2 illustrates the morphology of the global magnetic field in the four analyzed runs. The lines show the magnetic field lines which are connected to the spherical shell of $4000 \mathrm{~km}$ from the center of Mars. The morphology of the magnetic field in the analyzed four cases has been studied in detail earlier in Kallio et al. (2008). Here we focus on how the structure of the magnetic field helps us to understand the spatial distribution of the planetary oxygen ions.

In the non-magnetized case the magnetic field lines connected to the spherical shell form a magnetotail behind the planet. Roughly speaking, the magnetic field lines which starting points are on the spherical shell on the dusk side, which is shown in Fig. 2(a), form a magnetic tail lobe on the dusk side. Another magnetic tail lobe is in the dawn hemisphere (not visible in Fig. 2(a) because it is behind the $y=0$ color plane). Two different $\mathrm{O}_{2}{ }^{+}$regions can be identified in Fig. 2(a). First; $\mathrm{O}_{2}{ }^{+}$ions which are located near or within the magnetic tail lobes. The second high density region is on the Southern hemisphere where the convective electric field accelerates escaping planetary ions (see details in Kallio et al., 2010).

In the magnetized cases the magnetic field forms two regions which can be seen to correlate with the high density regions (Fig. 2(b-d)). First, the magnetic field forms a closed magnetic field line region where both ends of the magnetic field lines are connected to the spherical shell at $r=4000 \mathrm{~km}$. The volume inside the closed magnetic field line region increases and, similarly, the area of the closed magnetic field line on the surface of the spherical shell becomes larger with increasing dipolar field (see Kallio et al., 2008, Fig. 2, for details.). The high $\mathrm{O}_{2}{ }^{+}$density regions near the magnetic equator are, therefore, formed of $\mathrm{O}_{2}{ }^{+}$ ions inside the closed magnetic field lines.

The other magnetic region in the non-zero dipole cases is the open magnetic field line regions near the magnetic North and South poles. The magnetic field lines in these "magnetic cusps" are open, that is, only one end of the field line is connected to the spherical shell and the another end is connected to the faces of the simulation box at $x, y, z= \pm 6$ $R_{\mathrm{M}}$. The increased size of the non-zero density $\mathrm{O}_{2}{ }^{+}$regions in the Northern and Southern hemispheres is, therefore, related to the increased size of the magnetic cusps.

A third $\mathrm{O}_{2}{ }^{+}$density region which is also related to the magnetic field is the high density region on the Southern hemisphere in the solar wind far from Mars at $x>1 R_{\mathrm{M}}$. This region is related to the open magnetic field lines with one endconnected to the Southern hemisphere and the other end to the interplanetary magnetic field on the dayside (the magnetic field lines are not shown). The origin of this magnetic connection resembles what was earlier detected in the HYB-Mercury hybrid model run made for Mercury which has a weak intrinsic magnetic field (Kallio and Janhunen, 2003). In this simulation the positive IMF $x$-component connects IMF to the Southern hemisphere of Mercury. The magnetic connection of the intrinsic magnetic field and the IMF appears to enable leakage of $\mathrm{O}_{2}{ }^{+}$ions from Mars into the Solar wind.

\subsection{Motion of the escaping ions}

The correlation of the $\mathrm{O}_{2}{ }^{+}$density regions to the magnetic field suggests that the flow of planetary ions is related to the morphology of the magnetic field. This relation is studied in detail in Fig. 3 which shows the streamlines of 

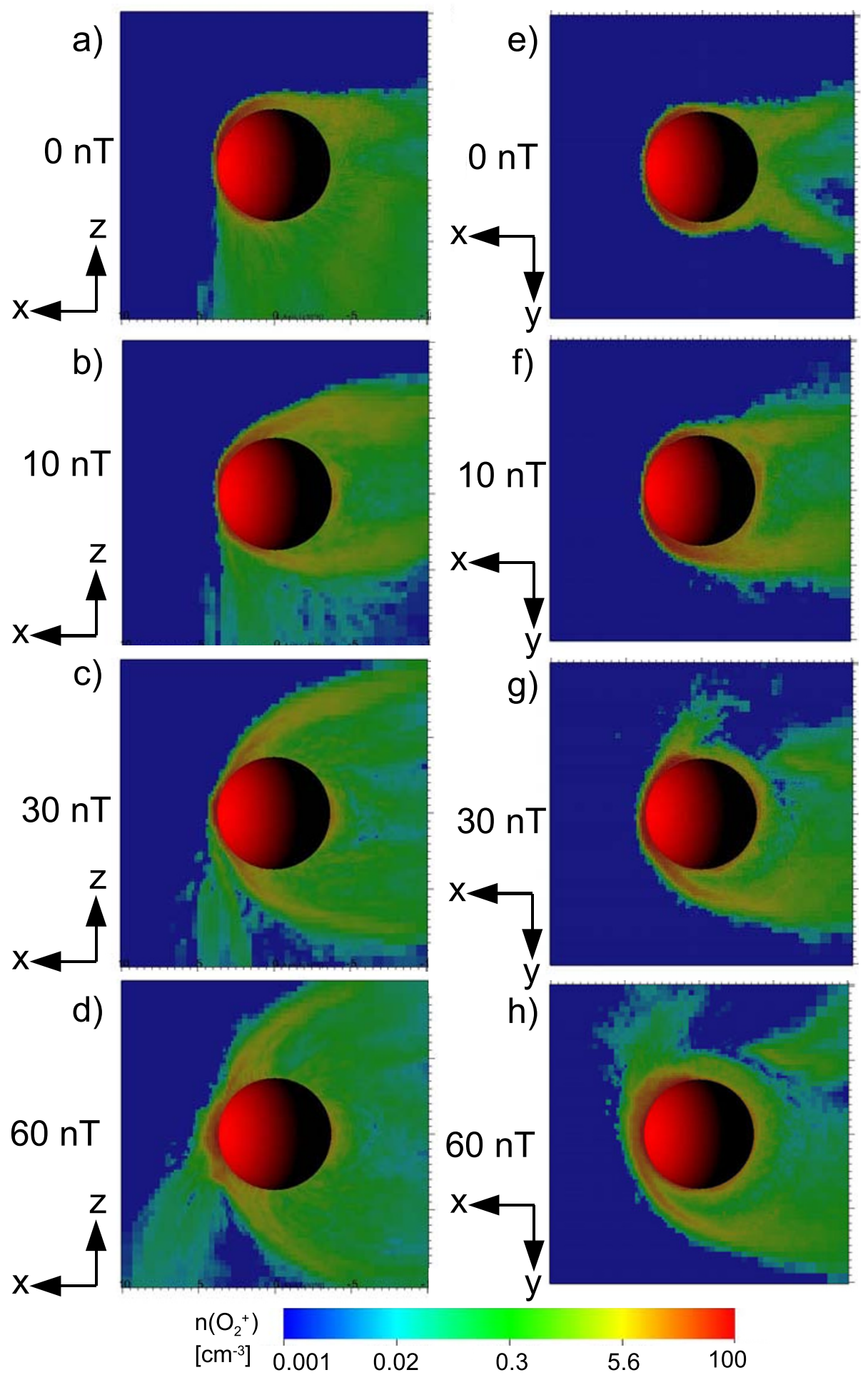

Fig. 1. (a-d) The density of $\mathrm{O}_{2}{ }^{+}$ions near Mars on the noon-midnight meridian plane $(y=0)$ and $(\mathrm{e}-\mathrm{h})$ on the equator plane $(z=0)$. The Martian intrinsic dipolar magnetic field is (a, e) $0 \mathrm{nT},(b, f) 10 \mathrm{nT},(\mathrm{c}, \mathrm{g}) 30 \mathrm{nT}$ and $(\mathrm{d}, \mathrm{h}) 60 \mathrm{nT}$ on the surface of Mars in the magnetic equator. The solar wind flows in the figures from left to right. In panels (a)-(d) the upper $(z>0)$ and the lower $(z<0)$ hemisphere is the North and the South hemisphere, respectively. In panels $(\mathrm{e})-(\mathrm{h})$ the upper $(y<0)$ and the lower $(y>0)$ hemisphere are the dawn and dusk hemispheres, respectively. The red sphere has a radius of $3700 \mathrm{~km}\left(\sim 1.09 R_{\mathrm{M}}\right)$.

$\mathrm{O}_{2}{ }^{+}$ions near Mars. The streamline tracing was started on the spherical shell of $4000 \mathrm{~km}$ from the center of Mars. Figure 3 also shows that in the non-magnetized case part of the $\mathrm{O}_{2}{ }^{+}$stream lines move to the nightside while some streamlines (associated with the "pick-up" $\mathrm{O}_{2}{ }^{+}$ions) have a notable velocity component in the $-z$ direction. In the magnetized cases the morphology of the $\mathrm{O}_{2}{ }^{+}$streamlines resembles closely the morphology of the open/closed magnetic field line regions (c.f. Fig. 2(b-d) and Fig. 3(b-d), $3(\mathrm{f}-\mathrm{h}))$. The density of $\mathrm{O}^{+}$ions has similar spatial distribution than the $\mathrm{O}_{2}{ }^{+}$ions with the exception that $\mathrm{O}^{+}$ions originating from the hot oxygen corona form spatially more 

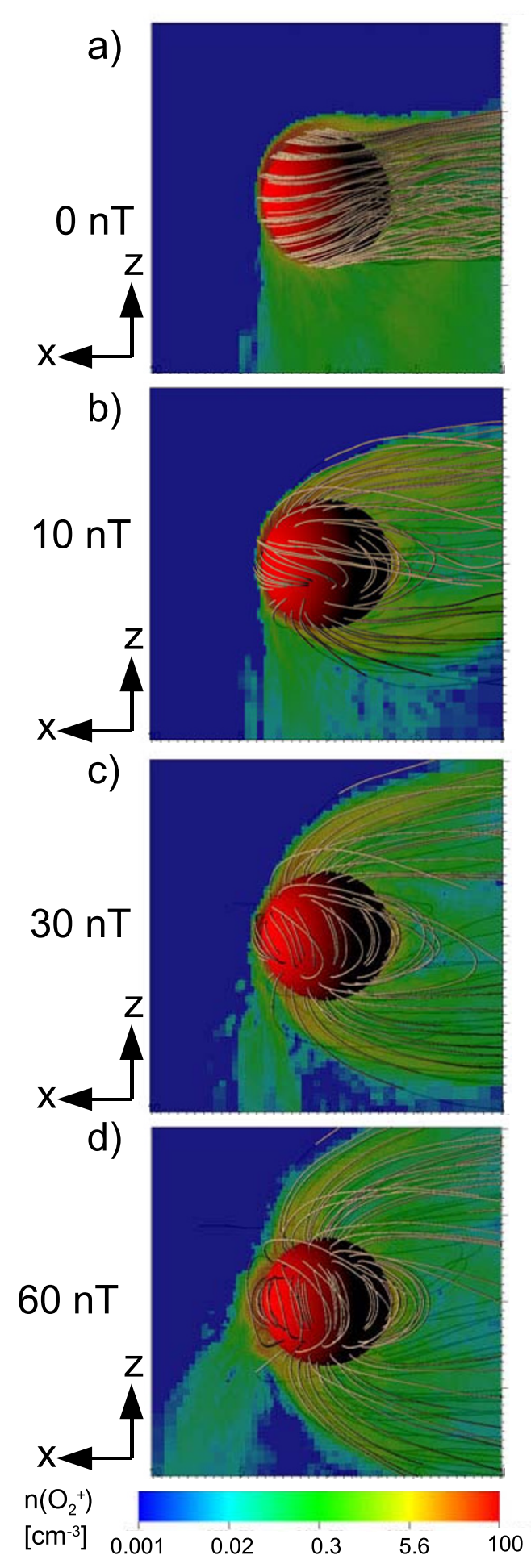

Fig. 2. Magnetic field lines near Mars when the Martian intrinsic dipolar magnetic field is (a) $0 \mathrm{nT}$, (b) $10 \mathrm{nT}$, (c) $30 \mathrm{nT}$ and (d) $60 \mathrm{nT}$ on the surface of Mars in the magnetic equator. The field line tracing was started on the spherical shell of $4000 \mathrm{~km}$ from the center of Mars. The color planes at the noon-midnight plane $(y=0)$ show the density of $\mathrm{O}_{2}+$ ions and they are added to see the correlation of density regions to the magnetic field lines. The red sphere has a radius of $3700 \mathrm{~km}$. Note that the colour of the magnetic field lines which are behind the $y=0$ colour plane is dark blue and they are not as bright as the field lines above the $y=0$ plane. extended density regions than $\mathrm{O}_{2}{ }^{+}$ions (Fig. 3(e-h)) which are originating purely from the model exobase near Mars. The $\mathrm{O}^{+}$and $\mathrm{O}_{2}{ }^{+}$streamline patterns are also very similar.

It is instructive to examine the $60 \mathrm{nT}$ case in greater detail and note the following. First, the $\mathrm{O}^{+}$and $\mathrm{O}_{2}+$ ions on the closed magnetic field line region have a sunward motion on the dusk side, that is, they are rotating around Mars counterclockwise when viewed from above the North Pole (Fig. 3(d) and 3(h)). This motion is probably related to the magnetic gradient and curvature drifts as is the case in the Earth's ring currents. The high density regions on the magnetic equator in the $60 \mathrm{nT}$ case may therefore be related to the ion drift motion which transports ions, at least partly, around the planet. Some of these drifting ions seem to become lost into the solar wind on the dawn side, maybe because of the asymmetries associated with the non-zero IMF $x$-component. The streamline in Fig. 3(d) (3(h)) also shows how the detached $\mathrm{O}_{2}{ }^{+}\left(\mathrm{O}^{+}\right)$enhancement on the dayside on the South hemisphere is related to the streamlines originating near the Southern magnetic cusp. Moreover, the velocity of $\mathrm{O}_{2}{ }^{+}$ions in the closed field line region is low, only few tens of $\mathrm{km} \mathrm{s}^{-1}$ (Fig. 4(a) and (b)). The velocity of ions in the cusp region, where the plasma escapes along the field lines, is much higher than ion velocities near the magnetic equator. Note that the small-scale circular structures on the spherical shell in Fig. 4(b) are artefacts caused by interpolation of the velocity values from a Cartesian grid onto a surface of a sphere.

It is instructive to estimate the role of the kinetic effects in the velocity values shown in Fig. 4. The lines shown earlier in Fig. 3 show the streamlines derived from the ion bulk velocity field. An ion which has a velocity component perpendicular to the magnetic field, $v_{\text {perp }}$, however, rotates around the magnetic field. For example, $\mathrm{a} \mathrm{O}_{2}{ }^{+}$ion in the 10 nT magnetic field which has $v_{\text {perp }}$ of, say, $1 \mathrm{~km} \mathrm{~s}^{-1}, 8 \mathrm{~km}$ $\mathrm{s}^{-1}$ and $50 \mathrm{~km} \mathrm{~s}^{-1}$ has gyroradius of $\sim 30 \mathrm{~km}, \sim 300 \mathrm{~km}$, and $\sim 1700 \mathrm{~km}$, respectively. The two first gyroradii $(30 \mathrm{~km}$ and $300 \mathrm{~km}$ ) are much smaller than the radius of Mars. The last of these gyroradii $(1700 \mathrm{~km})$ starts to become comparable with the radius of Mars and is large compared with the interaction regions near the subsolar point. These sizes suggest that the trajectories of $\mathrm{O}_{2}{ }^{+}$ions which have velocity of several $\mathrm{km} \mathrm{s}^{-1}$ and above may differ noticeable from the flow lines. In a $60 \mathrm{nT}$ field, by contrast, the gyroradii are $\sim 5 \mathrm{~km}, \sim 40 \mathrm{~km}$ and $\sim 300 \mathrm{~km}$ for $v_{\text {perp }}$ of $1 \mathrm{~km} \mathrm{~s}^{-1}$, $8 \mathrm{~km} \mathrm{~s}^{-1}$ and $50 \mathrm{~km} \mathrm{~s}^{-1}$, respectively, which all are small compared with the radius of Mars.

3.4 Loss rates of $\mathrm{O}^{+}$and $\mathrm{O}_{2}^{+}$ions through the exobase

It is finally interesting to examine how the intrinsic magnetic field affects to the total loss rate of $\mathrm{O}^{+}$and $\mathrm{O}_{2}{ }^{+}$ions through the exobase in the analyzed runs. Table 1 shows the total loss rate of $\mathrm{O}^{+}$and $\mathrm{O}_{2}+$ ions formed in the ionosphere below the inner obstacle of the model. These ions are emitted in the model from the exobase at a constant rate of $1.4 \times 10^{25} \mathrm{~s}^{-1}\left(\mathrm{O}^{+}\right.$ions $)$and $2.0 \times 10^{25} \mathrm{~s}^{-1}\left(\mathrm{O}_{2}{ }^{+}\right.$ions $)$. It is worth noting that all ion loss fractions are less than $100 \%$, implying that in all cases there is a planetary ion reservoir at the exobase which could have escaped from the Mars but that they have not all been lost (Table 1, the 4th and 5th columns). For example, the $\mathrm{O}^{+}$loss percentage of 30 in 

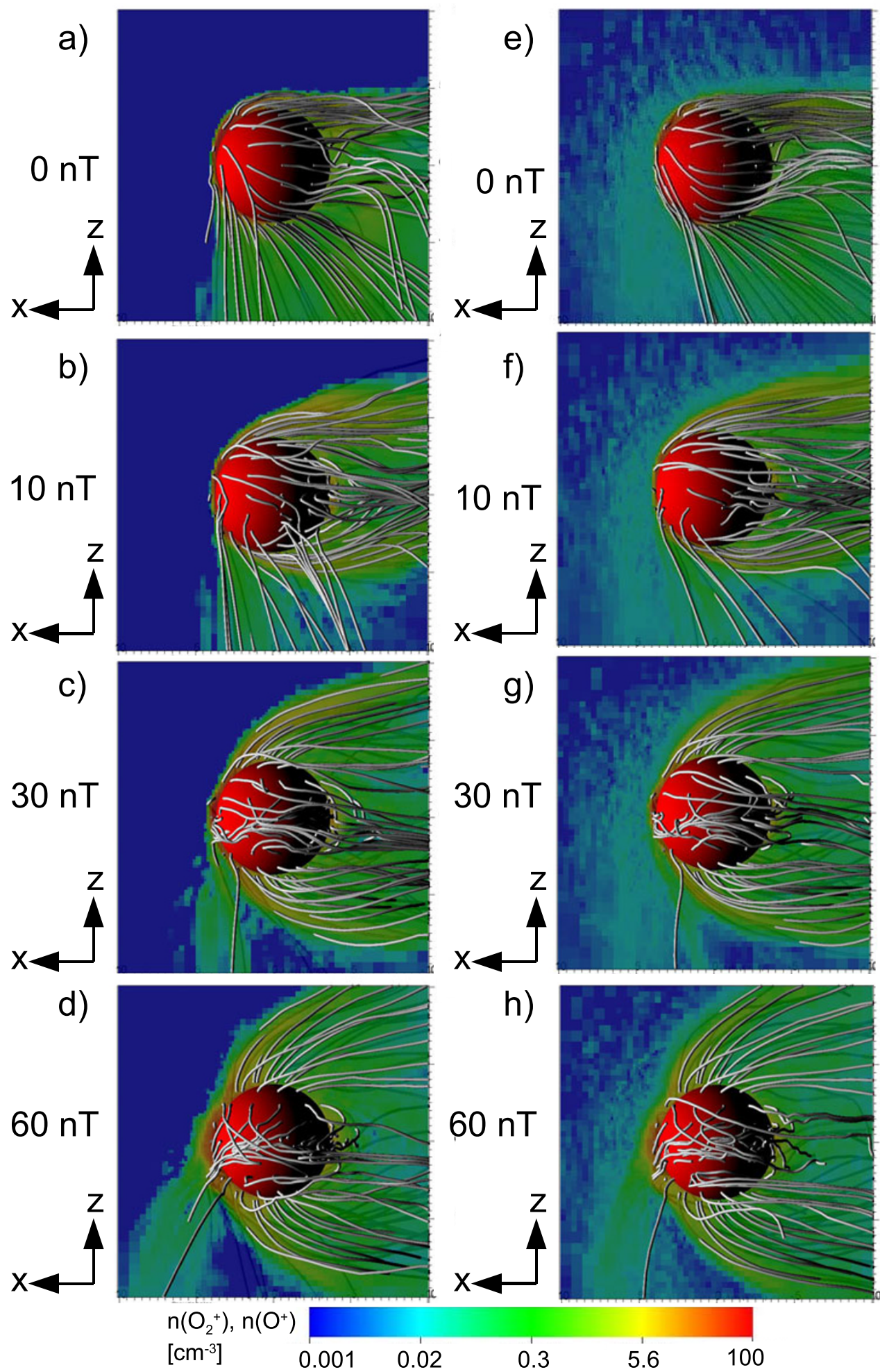

Fig. 3. Streamlines of the $\mathrm{O}_{2}{ }^{+}$ions (left column) and the $\mathrm{O}^{+}$ions (right column) near Mars when the Martian intrinsic dipolar magnetic field is (a, e) 0 $\mathrm{nT},(\mathrm{b}, \mathrm{f}) 10 \mathrm{nT},(\mathrm{c}, \mathrm{g}) 30 \mathrm{nT}$ and $(\mathrm{d}, \mathrm{h}) 60 \mathrm{nT}$ on the surface of Mars in the magnetic equator. The streamline tracing was started on the spherical shell of $4000 \mathrm{~km}$ from the center of Mars. The color planes at the noon-midnight plane $(y=0)$ show the ion density and they are added in order to see the correlation of the ion density regions to the stream lines. The red sphere has a radius of $3700 \mathrm{~km}\left(\sim 1.09 R_{\mathrm{M}}\right)$. Note that the colour of the magnetic field lines which are behind the $y=0$ colour plane is dark blue and they are not as bright as the field lines above the $y=0$ plane.

the $0 \mathrm{nT}$ dipole run (Table 1 , the 4 th column of the left) means that $30 \%$ of the available $\mathrm{O}^{+}$ions which are emitted from the model exobase escape from Mars while the rest $(70 \%)$ of the emitted $\mathrm{O}^{+}$ions are re-absorbed at the model exobase. This implies that in the analyzed runs, the plan- etary ionosphere ion loss is not "source limited" because only about every third of the emitted planetary ions from the exobase escapes from Mars while about two out of three emitted ions re-encounters back to the exobase.

The last two columns in Table 1 show how much-in rel- 
a)
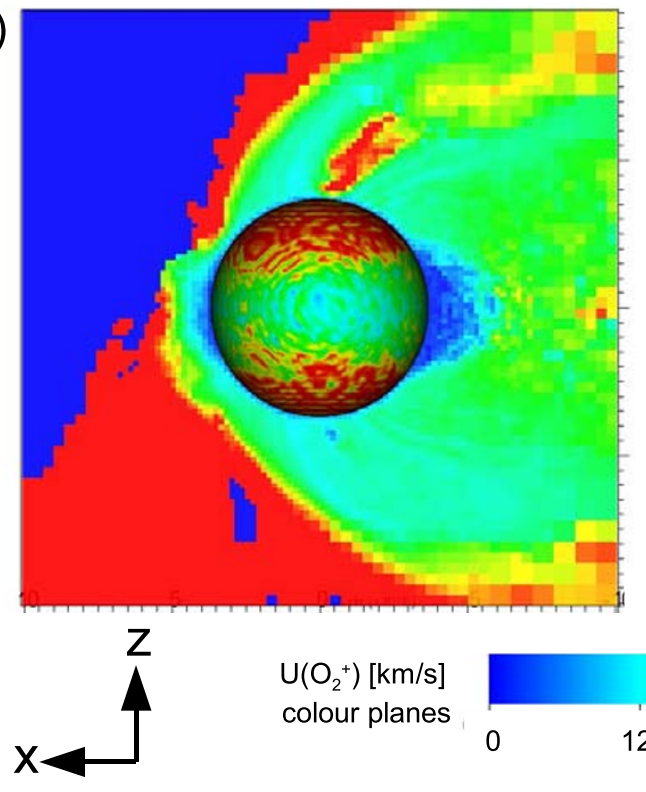

b)

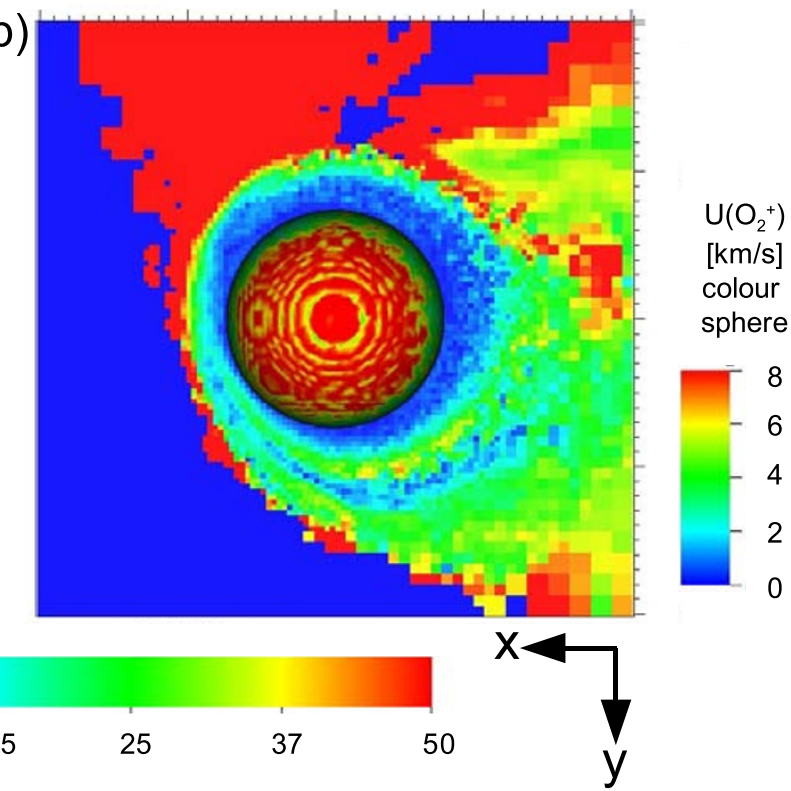

Fig. 4. The speed of $\mathrm{O}_{2}{ }^{+}$ions near Mars in the $60 \mathrm{nT}$ case. Note that the horizontal color map in the range $[0,50] \mathrm{km} \mathrm{s}{ }^{-1}$ shows the velocity on the $y=0$ plane (panel a) and on the $z=0$ plane (panel b) while the vertical color panel on the right in the velocity range [0,8] km $\mathrm{s}^{-1}$ gives the velocity on the spherical shell of the radius $3700 \mathrm{~km}$ just above the model exobase.

Table 1. The loss rate of $\mathrm{O}^{+}$and $\mathrm{O}_{2}{ }^{+}$ions through the exobase in the analyzed four runs with different Martian global intrinsic dipole fields.

\begin{tabular}{rcccccc}
\hline$B$ dipole & $\begin{array}{c}\mathrm{O}^{+} \text {loss rate } \\
\left(10^{24} \mathrm{~s}^{-1}\right)\end{array}$ & $\begin{array}{c}\mathrm{O}_{2}^{+} \text {loss rate } \\
\left(10^{24} \mathrm{~s}^{-1}\right)\end{array}$ & $\begin{array}{c}\mathrm{O}^{+} \\
\text {loss } \%\end{array}$ & $\begin{array}{c}\mathrm{O}_{2}^{+} \\
\text {loss } \%\end{array}$ & $\begin{array}{c}\mathrm{O}^{+} \text {loss } \\
\text { rate change }\end{array}$ & $\begin{array}{c}\mathrm{O}_{2}{ }^{+} \text {loss } \\
\text { rate change }\end{array}$ \\
\hline $0 \mathrm{nT}$ & 4.26 & 7.91 & 30 & 40 & $0 \%$ & $0 \%$ \\
$10 \mathrm{nT}$ & 5.76 & 8.61 & 41 & 43 & $+35 \%$ & $+9 \%$ \\
$30 \mathrm{nT}$ & 4.76 & 7.14 & 34 & 36 & $+12 \%$ & $-10 \%$ \\
$60 \mathrm{nT}$ & 4.27 & 6.09 & 31 & 30 & $+0.3 \%$ & $-23 \%$ \\
\hline
\end{tabular}

In all four runs the total ion emission rate from the exobase was $1.4 \times 10^{25} \mathrm{~s}^{-1}$ for $\mathrm{O}^{+}$ions and $2.0 \times 10^{25} \mathrm{~s}^{-1}{\text { for } \mathrm{O}_{2}+}^{+}$ ions. The seven columns from left to right are [1] the strength of the dipolar magnetic field on the surface of Mars in the magnetic equator, [2] the total loss rate of $\mathrm{O}^{+}$ions through the exobase, [3] the total loss rate of $\mathrm{O}_{2}+$ ions through the exobase, [4] the percentage of $\mathrm{O}^{+}$ions emitted from the exobase that are lost $\left(=\mathrm{O}^{+}\right.$loss rate $\left./ 1.4 \times 10^{25} \mathrm{~s}^{-1}\right)$, [5] the percentage of $\mathrm{O}_{2}+$ ions emitted from the exobase that are lost $\left(=\mathrm{O}_{2}{ }^{+}\right.$loss rate $\left./ 2.0 \times 10^{25} \mathrm{~s}^{-1}\right)$, [6] the $\mathrm{O}^{+}$loss rate change compared with the non-magnetized case $\left(=100 \times\left(\left(\mathrm{O}^{+} \operatorname{loss}\right.\right.\right.$ rate $\left.\left.\left.\left.-4.26 \times 10^{24} \mathrm{~s}^{-1}\right) / 4.26 \times 10^{24} \mathrm{~s}^{-1}\right) \%\right)\right)$ and [7] the $\mathrm{O}_{2}{ }^{+}$loss rate change compared with the non-magnetized case $\left(=100 \times\left(\left(\mathrm{O}_{2}{ }^{+}\right.\right.\right.$loss rate $\left.-7.91 \times 10^{24} \mathrm{~s}^{-1}\right) /$ $\left.7.91 \times 10^{24} \mathrm{~s}^{-1}\right) \%$ ).

ative terms - the dipolar magnetic field values have changed the total ion loss rates compared with the non-magnetized case. Interestingly, the highest ionosphere $\mathrm{O}^{+}$ion loss rate increase $(35 \%)$ and the highest ionosphere $\mathrm{O}_{2}{ }^{+}$ion loss rate increase $(9 \%)$ were obtained at the weakest $10 \mathrm{nT}$ intrinsic magnetic field situation and neither in the non-magnetized case nor in the highest magnetic field case of $60 \mathrm{nT}$. The streamlines of oxygen ions (cf. Fig. 3) suggest that this may be related to relative size of the open and closed magnetic field line regions. In the non-magnetized case the radial component of the magnetic field at the exobase is zero which limits the escape of ions along the magnetic field lines. The non-zero intrinsic magnetic field results in a nonzero radial magnetic field at the exobase enabling magnetic field aligned ion escape from the ionosphere through the magnetic cusps. But at the same time the increase of the dipolar magnetic field creates a closed magnetic field line region, inside which ions can be trapped. The increased magnetic field, therefore both increases the loss of ions in certain regions (magnetic cusps) and reduces it in other regions (a closed magnetic field line region). The maximum total ion loss rate is obtained in the situation where the magnetic cusps enable magnetic field aligned ion loss but where the intrinsic magnetic field has not yet formed very large and strong closed field line region.

\section{Discussion}

This paper presents the properties of $\mathrm{O}^{+}$and $\mathrm{O}_{2}{ }^{+}$ions near Mars in a situation where the planet has been assumed to have a weak global intrinsic magnetic field while the other input parameters (such as the properties of the solar wind, the IMF, neutral density profiles, and the EUV flux) correspond to the present day conditions at Mars (see discussion in Section 2.2).

The main new feature in the presented simulations is inclusion of a non-zero Martian intrinsic magnetic field, approximated by a dipole. Such a field configuration is, however, an overly simplified description for both current 
and past magnetic field of the planet. Estimates based on MGS magnetic field measurements have shown the maximum present day Martian magnetic dipole to be $\sim 2 \times 10^{17}$ $\mathrm{A} / \mathrm{m}^{2}$ (Acuña et al., 2001). Such a magnetic dipole produces only a field of $\sim 0.5 \mathrm{nT}$ at the equator on the surface implying that the current Mars has not a significant global dynamo-generated dipolar magnetic field. Although the magnetic field is strong in the southern hemisphere near the magnetic anomalies, they do not produce any noticeable dipolar field (see, for example, Connerney et al., 2004, for an overview of MGS magnetic field observations and their interpretations). On the other hand the strong crustal magnetic anomalies suggest that before the Martian dynamo ceased in the past, the dynamo generated magnetic field was much larger than the dipolar magnetic field values used in the simulation runs analysed in this paper. All these aspects have to be taken into account in the forthcoming more detailed young Mars-solar wind interaction studies.

Despite the aforementioned limitations of the hybrid model used here, the obtained results are foreseen to be represent the first step in estimating quantitatively how the global intrinsic magnetic field affects the properties of planetary ions at Mars. Next steps include more a detailed study of the effects of the dipole field not being perpendicular to the Mars-Sun line, of the crustal magnetic field, of the solar wind, of the IMF and of the EUV flux when Mars was young, when precipitating ions affect the emission of planetary ions from the exobase and, finally, taking into account self-consistently ionospheric processes.

\section{Summary}

We have studied the characteristics of the Martian $\mathrm{O}^{+}$and $\mathrm{O}_{2}{ }^{+}$plasma environment in cases where a small global intrinsic magnetic field has values of $10 \mathrm{nT}, 30 \mathrm{nT}$ and $60 \mathrm{nT}$. A few tens of nT surface magnetic field was found to result in two different high density plasma regions which are related to two different magnetic field regions, namely, (1) a slow velocity $\mathrm{O}^{+}$region on closed magnetic field lines and (2) a high velocity region on open magnetic field line region. The high density region on closed magnetic field lines was formed from planetary ions which cannot escape from the "magnetic bottle". At the cusps, however, the planetary ions can escape along open magnetic field lines. The relative size of the closed and open magnetic field line regions depends on the strength of the intrinsic magnetic field. Interestingly, the largest loss rate of ionospheric oxygen through the exobase was found in the $10 \mathrm{nT}$ case.

Acknowledgments. The authors would like to thank Drs. Riku Jarvinen and Pekka Janhunen at Finnish Meteorological Institute, for their valuable contribution in developing of the HYB-modeling platform. The authors like also thank Dr. Tero Siili for the careful inspection of the manuscript and the two referees for valuable suggestions and comments of to improve the manuscript. The figures are made by VisIt open source visualization tool.

\section{References}

Acuña, M. H. et al., Magnetic field and plasma observations at Mars: initial results of the Mars global surveyor mission, Science, 279, 1676-1680, 1998

Acuña, M. H. et al., The magnetic field of Mars: Summary of results from the aerobraking and mapping orbits, J. Geophys. Res., 106(E10), 23,403-23,418, 2001.

Barabash, S., A. Fedorov, R. Lundin, and J.-A. Sauvaud, Martian atmospheric erosion rates, Science, 315(5811), 501-503, doi:10.1126/science.1134358, 2007.

Brain, D. S. et al., A comparison of global models for the solar wind interaction with Mars, Icarus, 206, 139-151, doi:10. 1016/j.icarus.2009.06.030, 2010.

Connerney, J. E. P. et al., Mars crustal magnetism, Space Sci. Rev., 111, 1-33, 2004

Kallio, E. and P. Janhunen, Solar wind and magnetospheric ion impact on Mercury's surface, Geophys. Res. Lett., 30(17), 1877, doi:10.1029/2003GL017842, 2003.

Kallio, E., S. Barabash, P. Janhunen, and R. Jarvinen, Magnetized Mars: Transformation of Earth-like magnetosphere to Venuslike induced magnetosphere, Planet. Space Sci., 56(6), 823-827, doi:10.1016/j.pss.2007.12.005, 2008.

Kallio, E., K. Liu, R. Jarvinen, V. Pohjola, and P. Janhunen, Oxygen ion escape at Mars in a hybrid model: high energy and low energy ions, Icarus, doi:10.1016/j.icarus.2009.05.015, 2010.

Ledvina, S. A., Y.-J. Ma, and E. Kallio, Modeling and simulating flowing plasmas and related phenomena, Space Sci. Rev., doi:10.1007/s11214008-9384-6, 2008.

Moore, T. E. and G. V. Khazanov, Mechanisms of ionospheric mass escape, J. Geophys. Res., 115, A00J13, doi:10.1029/2009JA014905, 2010.

Moore, T. E., M.-C. Fok, D. C. Delcourt, S. P. Slinker, and J. A. Fedder, Global response to local ionospheric mass ejection, J. Geophys. Res., 115, A00J14, doi:10.1029/2010JA015640, 2010.

Ribas, I., E. F. Guinan, M. Gudel, and M. Audard, Evolution of the solar activity over time and effects on planetary atmospheres. I. High-energy irradiances (1-1700 ̊), Astrophys. J., 622, 680-694, 2005.

Wood, B. E., The solar wind and the Sun in the past, Space Sci. Rev., 126(1-4), 3-14, doi:10.1007/s11214-006-9006-0, 2006.

E. Kallio (e-mail: esa.kallio@fmi.fi) and S. Barabash 